\title{
A functional C-terminal TRAF3-binding site in MAVS participates in positive and negative regulation of the IFN antiviral response
}

Suzanne Paz ${ }^{1,2}$, Myriam Vilasco ${ }^{3}$, Steven J Werden ${ }^{1}$, Meztli Arguello ${ }^{1}$, Deshanthe Joseph-Pillai ${ }^{1,2}$, Tiejun Zhao ${ }^{1}$, Thi Lien-Anh Nguyen ${ }^{1}$, Qiang Sun ${ }^{1}$, Eliane F Meurs ${ }^{3}$, Rongtuan Lin ${ }^{1,4}$, John Hiscott ${ }^{1,2,4}$

${ }^{I}$ Terry Fox Molecular Oncology Group, Lady Davis Institute, Jewish General Hospital, Montreal, Quebec H3T1E2, Canada; ${ }^{2}$ Department of Microbiology and Immunology, McGill University Montreal, Quebec H3A 2B4, Canada; ${ }^{3}$ Department of Virology, Unit of Hepacivirus and Innate Immunity, Pasteur Institute, Paris 75724 France; ${ }^{4}$ Department of Medicine, McGill University Montreal, Quebec H3A 2B4, Canada

Recognition of viral RNA structures by the cytosolic sensor retinoic acid-inducible gene-I (RIG-I) results in the activation of signaling cascades that culminate with the generation of the type I interferon (IFN) antiviral response. Onset of antiviral and inflammatory responses to viral pathogens necessitates the regulated spatiotemporal recruitment of signaling adapters, kinases and transcriptional proteins to the mitochondrial antiviral signaling protein (MAVS). We previously demonstrated that the serine/threonine kinase IKKe is recruited to the C-terminal region of MAVS following Sendai or vesicular stomatitis virus (VSV) infection, mediated by Lys63-linked polyubiquitination of MAVS at Lys500, resulting in inhibition of downstream IFN signaling (Paz et al, Mol Cell Biol, 2009). In this study, we demonstrate that C-terminus of MAVS harbors a novel TRAF3-binding site in the aa450468 region of MAVS. A consensus TRAF-interacting motif (TIM), 455-PEENEY-460, within this site is required for TRAF3 binding and activation of IFN antiviral response genes, whereas mutation of the TIM eliminates TRAF3 binding and the downstream IFN response. Reconstitution of $\mathrm{MAVS}^{-/}$mouse embryo fibroblasts with a construct expressing a TIM-mutated version of MAVS failed to restore the antiviral response or block VSV replication, whereas wild-type MAVS reconstituted antiviral inhibition of VSV replication. Furthermore, recruitment of IKKe to an adjacent C-terminal site (aa 468-540) in MAVS via Lys500 ubiquitination decreased TRAF3 binding and protein stability, thus contributing to IKKE-mediated shutdown of the IFN response. This study demonstrates that MAVS harbors a functional C-terminal TRAF3-binding site that participates in positive and negative regulation of the IFN antiviral response.

Keywords: MAVS; TRAF3; IKKe; IFN signaling; RIG-I Signaling

Cell Research (2011) 21:895-910. doi:10.1038/cr.2011.2; published online 4 January 2011

\section{Introduction}

To mount an effective antiviral response, the immune system must trigger multiple signaling pathways to promote the production of cytokines and other antiviral factors that collectively suppress viral replication and

Correspondence: John Hiscott

Tel: 514-340-8222, ext. 5265; Fax: 514-340-7576

E-mail: john.hiscott@mcgill.ca

Received 9 July 2010; revised 25 October 2010; accepted 8 November 2010; published online 4 January 2011 assembly [1]. The most robust antiviral response can be attributed to the induction of type I interferons (IFN) - IFN $\alpha$ and IFN $\beta$. On immunological assault, cellular pattern recognition receptors are rapidly stimulated following the recognition of specific molecular signatures of pathogens, which are often referred to as pathogenassociated molecular patterns $[2,3]$. In the case of an infection by RNA viruses and some DNA viruses, both the membrane-bound Toll-like receptors (TLRs) and cytosolic sensors, such as retinoic acid-inducible gene-I (RIG-I)-like receptors (RLRs), have been clearly shown to detect $5^{\prime}$ triphosphate-containing viral RNA structures, 
generated as a consequence of early virus transcription and/or replication $[3,4]$. Consequently, detection of viral RNA triggers the rapid activation of various transcription factors, such as NF- $\mathrm{kB}, \mathrm{AP}-1$ and members of the IFN regulatory factor (IRF) family (IRF1/3/7), that specifically bind to the IFN $\beta$ promoter to stimulate gene expression. Following production, IFN $\beta$ engages its cognate receptor and induces a complex intracellular signaling process to specifically activate the expression of multiple IFN-stimulated genes (ISGs), all of which contain a variation of the DNA sequence referred to as IFN-stimulated response element [5].

RLRs including RIG-I, melanoma differentiation factor 5 (MDA5) and laboratory of genetics and physiology 2 (LGP2) reside in the cellular cytoplasm, whereas TLRs are located on the plasma membrane or at the endosomal surface [2, 6-9]. Structurally, all three RLRs contain a DExD-box RNA helicase domain for RNA binding and with the exception of LGP2, also possess a caspase recruitment domain (CARD) that mediates downstream protein-protein interactions. Upon viral RNA recognition, activation of RIG-I promotes self-dimerization and other structural modifications that permit CARD-CARD interaction with the downstream adapter molecule mitochondrial antiviral signaling protein (MAVS; also known as IPS-1/Cardif/VISA) [10-13]. MAVS is composed of multiple motifs including: a C-terminal transmembrane domain (TM), which is essential for targeting to the outer mitochondrial membrane, three TRAF-interacting motifs (TIM), two included in the N-terminal proline-rich region (Pro), and an N-terminal CARD domain. Functionally, the CARD and TM domains appear to be critical for MAVS dimerization and for relaying the signal from RIG-I to downstream adapter molecules [12, 14-17]. The Pro region harbors two distinct TIMs, one located at aa 143-PVQET-147 that binds TRAF2 and TRAF3, and a second TIM located at aa 153-PGENSE-158 that exclusively binds TRAF6 $[13,18]$. An alternate TRAF6binding site is located in the C-terminus of MAVS at aa 455-PEENEY-460 [13], and both N- and C-terminal sites are required for TRAF6-mediated activation of the NF$\kappa \mathrm{B}$ pathway [13]. Additionally, MAVS interacts with multiple proteins, including LGP2, TRADD, FADD, RIP1, TRAF5, IKK $\varepsilon$, caspase 8/9 and MITA/STING/ MYPS/ERIS, TOM70, NLRX1, PCBP2/AIP4, OPTN, PLK1, deubiquitination enzyme A (DUBA), A20, [9$11,15,19-32]$, all indicating that MAVS functions as a molecular scaffold to which key adapter proteins bind to orchestrate the RIG-I dependent antiviral response.

TRAF family members are primarily involved in the regulation of inflammation, antiviral responses and apoptosis, and function downstream of the TNF, CD40 and
LT $\beta R$ receptors, to name a few. TRAF proteins play nonoverlapping roles in signaling; e.g., TRAF3 uniquely regulates the type I IFN response [33-35], while both TRAF2 and TRAF6 activate the NF- $\kappa$ B pathway downstream of RIG-I [13]. Thus, the RIG-I pathway bifurcates at the level of the TRAFs into two distinct pathways. TRAF2 and TRAF6 activate the classical IKK $\alpha / \beta$ kinases, and induce phosphorylation of I $\mathrm{B} \mathrm{B} \alpha$ inhibitor, resulting its proteasomal degradation. Active p65/p50 $\mathrm{NF}-\kappa \mathrm{B}$ dimers are then released and translocate into the nucleus to activate NF- $\mathrm{KB}$-dependent target genes [3639]. TRAF3 stimulates the non-canonical IKK-related kinases, TBK1 and IKKe, which induce C-terminal phosphorylation of IRF3/IRF7, leading to IRF3 dimerization, nuclear translocation, DNA binding and activation of IRF-dependent antiviral genes [26, 40-43].

Cytokine signal transduction is a transient process that is tightly regulated to prevent inappropriate inflammatory or autoimmune responses. Dynamic control of the antiviral response is coordinated at transcriptional levels, as well as by post-translational modifications, such as phosphorylation and ubiquitination. Multiple proteins are involved in the coordination of the RIG-I pathway including A20, DUBA, CYLD, NLRX1, OPTN, PCBP2-AIP4, PSMA7, miR146a, LGP2, NLRC5, gC1qR, FLN29, ISG15, RNF125, Pin1, SIKE and TOM70, to name a few [19, 27-28, 30, 32, 44-58]. Previously, we demonstrated that IKKe, but not TBK1, was recruited to the MAVS adapter via a Lys63-linked polyubiquitination on Lys500, thus revealing an unexpected function of IKKE in the negative regulation of the inflammatory and antiviral response [15]. We also identified Triad3A as an E3 ubiquitin ligase involved in the termination of the IFN response following viral infection, by targeting TRAF3 for K48linked ubiquitination and proteasomal degradation [59]. Recently, Tseng et al. [60] demonstrated that TRAF3 can either positively or negatively control the expression of type I IFN and pro-inflammatory cytokines through distinct types of ubiquitination.

Elucidating the spatiotemporal events involved in the recruitment of adapters, kinases and transcription factors to MAVS is essential for an understanding of the inflammatory and antiviral response. Although TRAF3 is essential for the IFN response, Seth et al. previously demonstrated that removal of the Pro domain from MAVS had no effect on IFN signaling, suggesting the presence of an alternate functional TRAF3-binding site. In this study, we identify a novel TRAF3-binding site in the Cterminus of MAVS (455-PEENEY-460) that is essential for MAVS-mediated antiviral responses. Furthermore, IKK $\varepsilon$, recruited to MAVS via K63-mediated ubiquitination of Lys500, decreases the binding and protein stabil- 
ity of TRAF3, thus contributing to negative regulation of the antiviral response.

\section{Results}

TRAF3 binds to a functional site within the C-terminus of MAVS

TRAF3 is an essential regulator of type I IFN expression following viral infection [35], with TRAF3 binding thought to be localized within the Pro region of MAVS [18]. However, removal of the Pro region had no effect on type I IFN production [12], implying the presence of another site. Therefore, we initially examined the ability of TRAF3 to interact with either the N-terminal, C-terminal or internal regions of MAVS $[12,13]$ (Figure 1A). The N-terminal constructs, 1-155 (MAVS aa 1-155); 1-400 (MAVS aa 1-400); or internal construct 157-400 (MAVS aa 157-400) of MAVS were not capable of bind-

\section{A}

TRAF3 Interaction

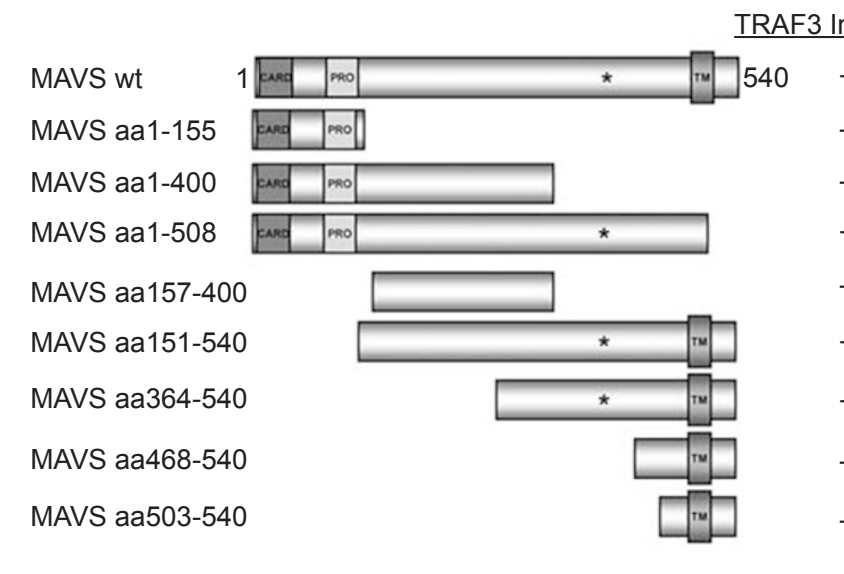

B

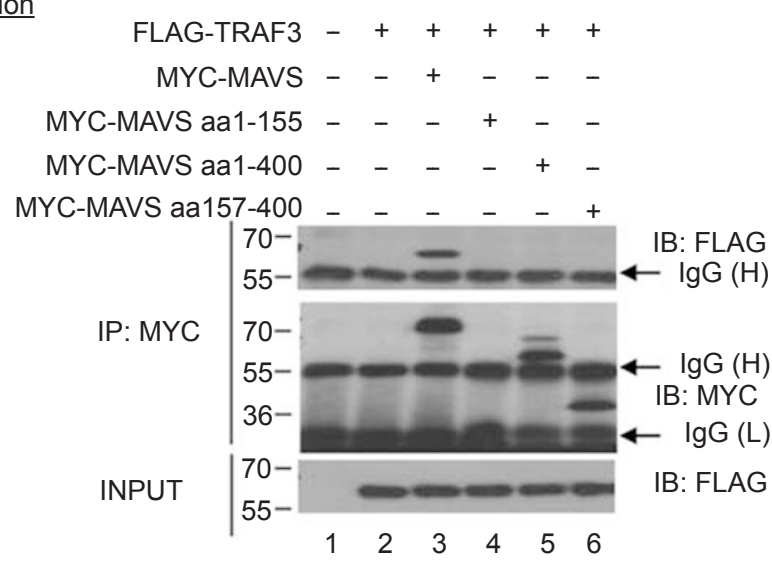

C
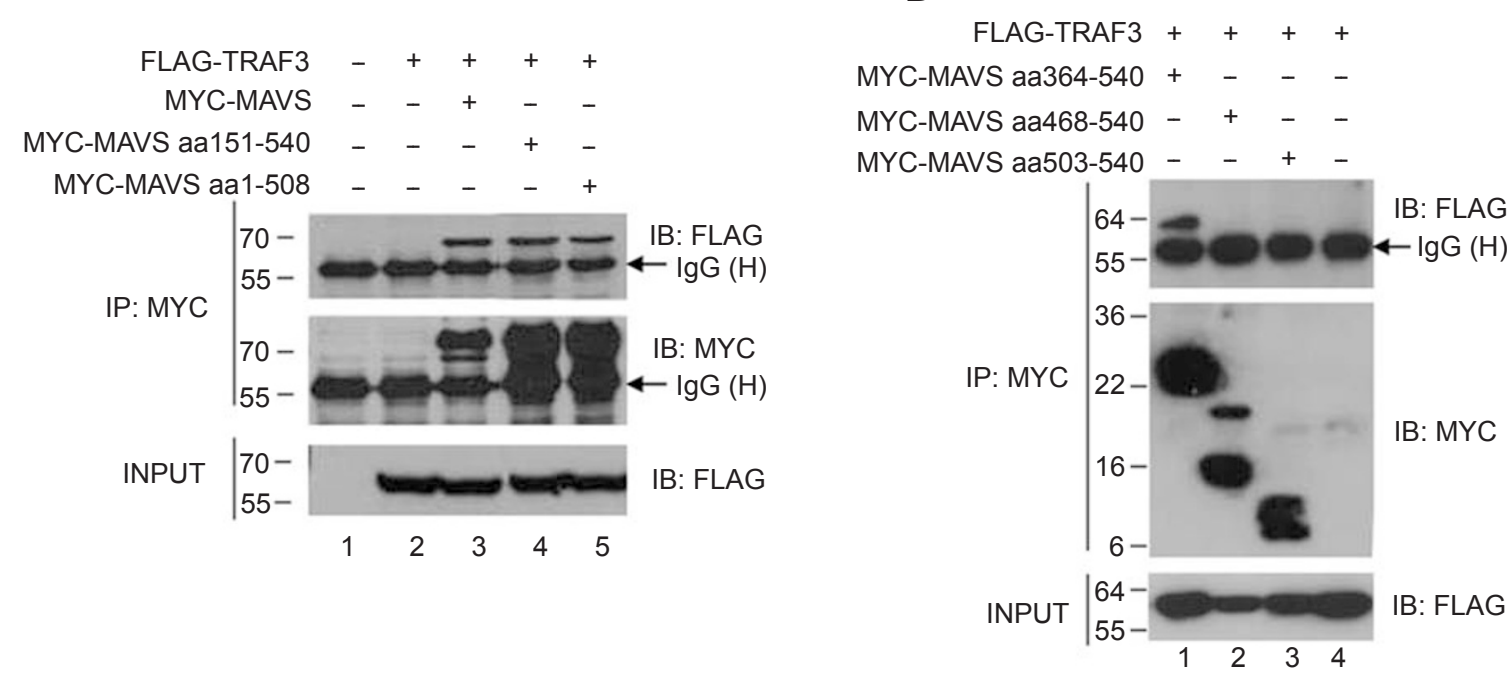

Figure 1 TRAF3 interacts with the C-terminus of MAVS. (A) Schematic representation of full-length MAVS and deletion constructs: MAVS aa 1-155, MAVS aa 1-400, MAVS aa 1-508, MAVS aa 157-400, MAVS aa 151-540, MAVS aa 364-540, MAVS aa 468-540 and MAVS aa 503-540. The location of the caspase recruitment domain (CARD), proline-rich region (Pro) and transmembrane domain (TM) are shown. The star is representative of the C-terminal TRAF-interacting motif (TIM). TRAF3 interaction profile to the various MAVS construct is depicted as plus (+) or minus (-) signs. (B-D) HEK293 cells were transfected with the indicated MAVS constructs. Co-immunoprecipitation was performed using an anti-MYC antibody (MAVS) followed by immunoblot with an anti-FLAG (TRAF3) antibody (top panel). Immunoprecipitated MAVS were revealed by immunoblot with anti-MYC antibody (second panel). Input for FLAG-TRAF3 is shown (bottom panel). 
ing TRAF3 when compared with MAVS wt (Figure 1B, compare lanes 4, 5 and 6 with lane 3), suggesting that the N-terminal or the internal region of MAVS was not capable of interacting with TRAF3 (Figure 1B). Deletion of the first 150 aa of MAVS, as in MAVS aa 151-540, did not reduce TRAF3-MAVS interaction (Figure 1A and $1 \mathrm{C}$, compare lane 4 with 3 ), further suggesting that $\mathrm{N}$-terminus of MAVS was dispensable for TRAF3 binding. Furthermore, removal of region aa 509-540, which includes the TM domain of MAVS (as in aa 1-508), did not affect binding to TRAF3 (Figure 1C, compare lane 5 with 3 ), indicating that the TM is not necessary for MAVS-TRAF3 interaction. A series of C-terminal MAVS constructs were used to demonstrate that TRAF3 binds to the C-terminal domain of MAVS: region aa 364-540 (Figure 1D, lane 1) but not region aa 468-540 or aa 503540 (Figure 1D, lanes 2 and 3) was capable of binding MAVS.

To further delineate the site of TRAF3 interaction, two different internally deleted MAVS constructs were coexpressed together with TRAF3 - MAVS $\triangle$ aa 101-450 and MAVS $\triangle$ aa 101-480. Both constructs lack the Pro region of MAVS, retain the CARD and TM, but differ in the size of the internal deletion that removes the C-terminal TIM motif (455-PEENEY-460; Figure 2A). TRAF3 interacted with MAVS $\triangle$ aa 101-450 but not with MAVS $\triangle$ aa 101-480, thus reducing the minimal site of TRAF3 interaction to aa 450-468 (Figure 2B compare lanes 2 and 3). To determine if this interaction was important for the antiviral response, quantitative PCR (qPCR) analysis of various genes induced following viral infection, $I F N B 1$, IFIT1 (ISG56), IP-10, OAS1 and RIG-I, was performed. On the basis of the qPCR results, MAVS $\triangle$ aa $101-450$ induced $I F N$ response genes similarly to MAVS wt, whereas a $50 \%$ decrease in gene expression was observed with MAVS $\triangle$ aa 101-480 (Figure 2C).

The C-terminal TIM of MAVS has been shown to bind TRAF6 [13], and although previous studies demonstrated that TRAF6 did not function in IFN signaling $[13,61]$, we sought to confirm that the activity of MAVS $\triangle$ aa 101-450 was mediated by TRAF3 and not TRAF6. In $\mathrm{TRAF}^{+/+}$and $\mathrm{TRAF}^{-/-}$MEFs, the MAVS $\triangle$ aa 101450 construct induced IFNa4 activity, both in the presence or absence of TRAF6, whereas MAVS $\triangle$ aa 101-480 did not activate the IFN $\alpha 4$ promoter in either TRAF $6^{+/+}$ or TRAF6 ${ }^{-1}$ MEFs (Figure 2D), thus ruling out the involvement of TRAF6 in MAVS $\triangle$ aa 101-450-driven activity. In contrast, when IFN induction was examined in TRAF3 $3^{-/}$MEFs ( $100^{-/-}$background), the ability of MAVS $\triangle$ aa 101-450 to initiate IFN transcription was reduced compared with TRAF ${ }^{+/+}$MEFs (Figure 2E). Together, these results demonstrate that MAVS activation of IFN signaling is regulated by TRAF3 binding to the C-terminal TIM of MAVS and does not require TRAF6 or the N-terminal Pro domain of MAVS.

TRAF3 association with the C-terminal TIM of MAVS is essential for IFN signaling

Next, the TIM site located in the C-terminus of MAVS was mutated from 455-PEENEY-460 to 455-AAANEY-460 (referred to as MAVS-Cterm AAA; Figure 3A). Interaction between MAVS wt and TRAF3 wt was readily detected in co-immunoprecipitation (Figure 3B, lane 3), whereas the interaction between TRAF3 and MAVS-Cterm AAA was reduced to undetectable levels (Figure 3B, lane 4). Subsequently, the ability of MAVS wt and MAVS-Cterm AAA to stimulate mRNA expression of various ISGs, IFNB1, IFNA2, IFIT1 (ISG56), IL6, and $I P-10$, was evaluated in HeLa cells (Figure 3C). Expression of MAVS wt stimulated mRNA levels for all ISGs, whereas MAVS-Cterm AAA did not induce ISG mRNA, or the activity of IFN $\alpha 4-$, IFN $\beta$-,

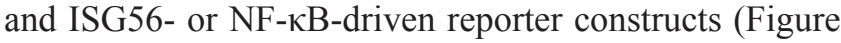
$3 \mathrm{C}$ and 3D). Furthermore, mutation of TRAF3 at amino acids Y440 and Q442 (TRAF3 Y440A/Q442A), residues critical for binding to the TIM of MAVS [18, 59], abrogated the interaction between TRAF3 Y440A/Q442A and MAVS (Figure 3E, lane 5), as well as MAVS-mediated IFN 44 promoter activity (Figure $3 \mathrm{~F}$ ). Thus, mutagenesis of the C-terminal TIM in MAVS or the TIM-interacting motif in TRAF3 eliminated both MAVS-TRAF3 interaction and downstream signaling to IFN response genes.

\section{$N$-terminal binding of TRAF3 to MAVS is not required} for IFN activation

To further clarify the discrepancy between $\mathrm{N}$ - versus C-terminal association of TRAF3, a MAVS expression construct was generated that mutated the N-terminal TIM at aa 143-PVQET-147 to aa 143-AVAEA-147 (referred to as MAVS-Nterm AAA; Supplementary information, Figure S1). Strikingly, mutation of the N-terminal TIM domain did not affect TRAF3 binding to MAVS (Supplementary information, Figure S1, lanes 3 and 4), and furthermore had no effect on IFN signaling, since ISG mRNA levels for IFNB1, IFNA2, IFIT1 (ISG56), IL6 and $I P-10$ remained similar in cells expressing MAVS or MAVS-Nterm AAA (Supplementary information, Figure S1). Additionally, MAVS-Nterm AAA retained the same functional activity as MAVS wt when monitored for

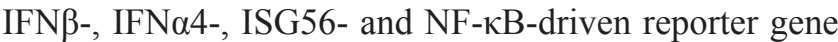
activity (Supplementary information, Figure S1). Thus, mutation of the N-terminal TIM did not affect TRAF3 binding or functional activity of MAVS, indicating that the N-terminal TIM does not have a role in TRAF3- 
A

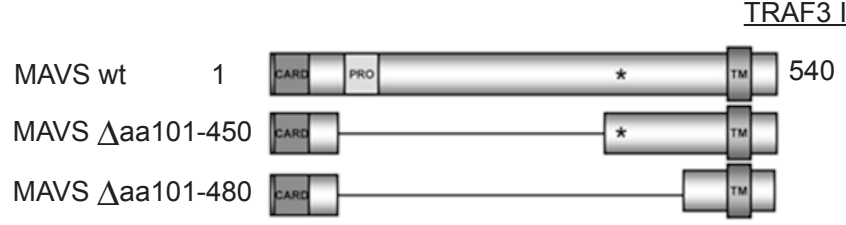

B

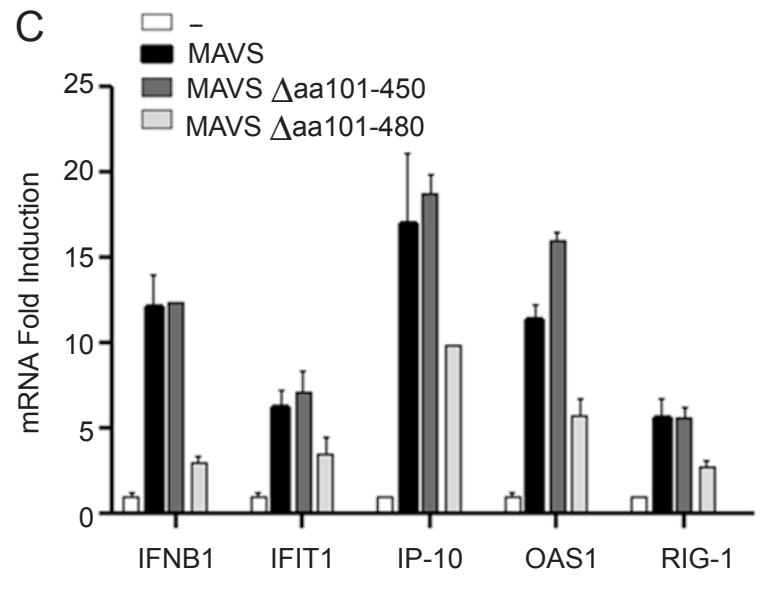

D

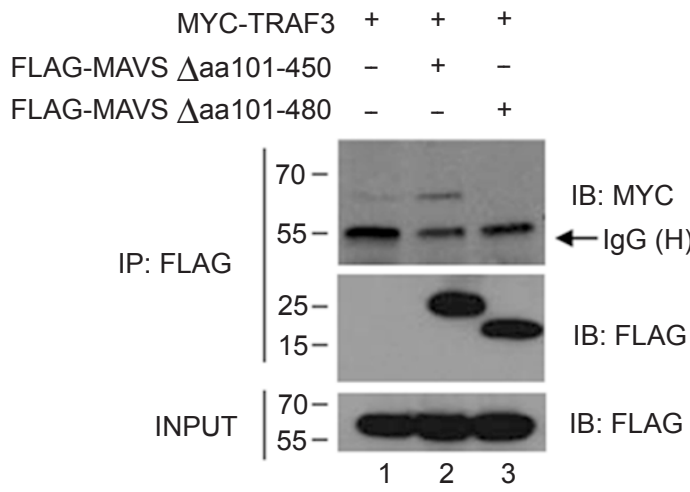

E

IFN $\alpha 4-L u c+$ IRF7
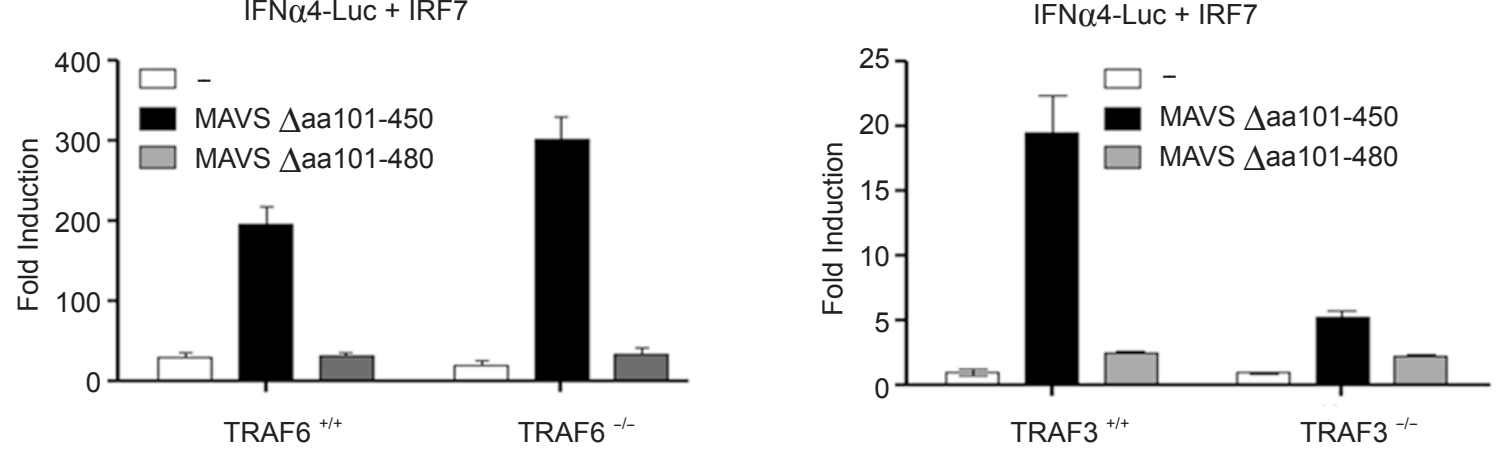

Figure 2 TRAF3 interacts with C-terminal region aa 450-468 of MAVS. (A) Schematic representation of full-length MAVS and internal deletion constructs: MAVS $\triangle$ aa101-450 and MAVS $\triangle$ aa101-480. The location of the Caspase recruitment domain (CARD), proline-rich region (Pro) and transmembrane domain (TM) are shown. The star is representative of the C-terminal TRAF-interacting motif (TIM). TRAF3 interaction profile to the various MAVS construct is depicted as plus $(+)$ or minus $(-)$ signs. (B) HEK293 cells were transfected with the indicated MAVS construct and TRAF3. Co-immunoprecipitation of MAVS was performed using anti-FLAG antibody followed with immunoblot with anti-MYC (TRAF3) antibody (top panel) or anti-FLAG (MAVS) antibody (second panel). Input for MYC-TRAF3 is shown (bottom panel). (C) qPCR analysis of total RNA isolated from HeLa cells transfected either with empty vector, MAVS wt, MAVS $\Delta$ aa101-450 or MAVS $\Delta$ aa101-480. Relative fold expression levels of IFNB1, IFIT1 (ISG56), IP-10, OAS1, and RIG-I versus ACTIN mRNA are shown. Data is representative of at least two experiments run in duplicate. (D-E) TRAF6 ${ }^{+/}$and TRAF6 ${ }^{-/-}$MEF cells (D) and TRAF3 $^{+/+}$and TRAF3 ${ }^{-/-}$MEFs (in a $^{2}$ p100 ${ }^{-1-}$ background) (E) were transfected with IFN $\alpha 4-L u c$ + IRF7 along with the expression plasmids encoding either empty vector, MAVS $\triangle$ aa101-450 or MAVS $\Delta$ aa101-480. Luciferase activity was measured at 24 h post transfection by Dual-Luciferase reporter assay as described by the manufacturer (Promega). Relative luciferase activity was measured as activation ( $n$-fold; relative to basal level of reporter gene in the presence of empty vector after normalization with co-transfected renilla luciferase activity); values are averages \pm standard deviations from three experiments. 

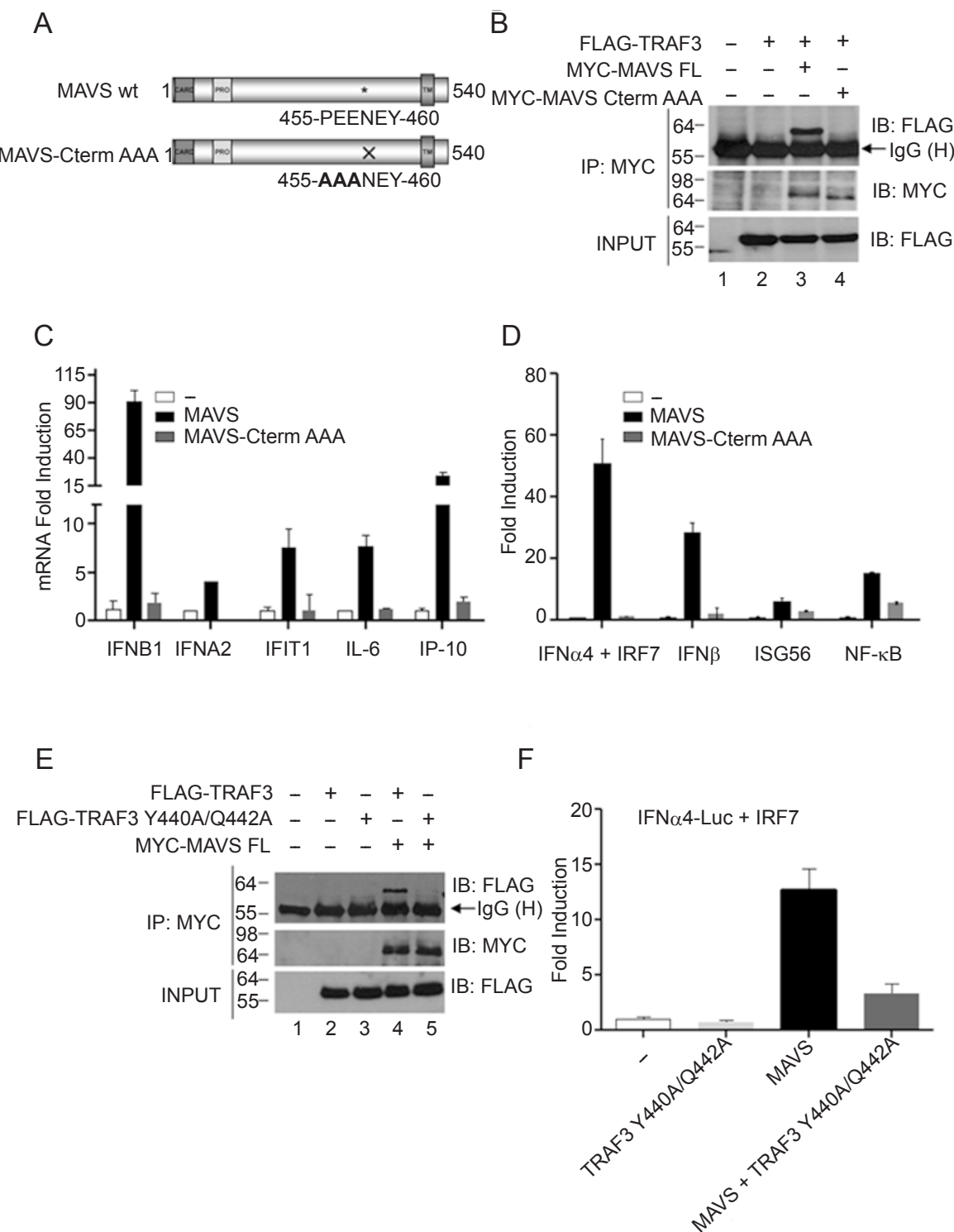

Figure 3 A MAVS C-terminal TRAF3-binding site is essential for the antiviral response. (A) Schematic representation of the MAVS wt and MAVS-Cterm AAA. The highly conserved sequence in region 455-PEENEY-460 of MAVS, as well as the mutations sites of MAVS-Cterm AAA is shown. The CARD, Pro and TM domains are also shown. (B) HEK293 cells were transfected with either: empty vector, FLAG-TRAF3, MYC-MAVS wt or MYC-MAVS-Cterm AAA alone or in co-transfection with FLAG-TRAF3. Co-immunoprecipitation was performed using an anti-MYC antibody followed by immunoblot with an anti-FLAG to reveal interaction with FLAG-TRAF3 (top panel). Immunoprecipitated MAVS was revealed by immunoblot with anti-MYC antibody (second panel). Input for TRAF3 is shown (bottom panel). (C) qPCR analysis of total RNA isolated from HeLa cells transfected either with empty vector, MAVS wt or MAVS-Cterm AAA. Relative fold expression levels of IFNB1, IFNA2, IFIT1 (ISG56), IL6 and IP-10 versus ACTIN mRNA are shown. Data is representative of at least two experiments run

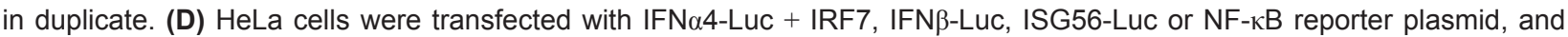
expression plasmids encoding either: empty, MAVS wt or MAVS-Cterm AAA as indicated. Luciferase activity was analyzed at $24 \mathrm{~h}$ post transfection and fold activation was determined compared with empty vector; values represent the average \pm S.D. Results are representative of at least three experiments run in triplicate. (E) HEK293 cells were transfected with either empty vector, FLAG-TRAF3, FLAG-TRAF3 Y440A/Q442A alone or in co-transfection with MYC-MAVS. Co-immunoprecipitation was performed using an anti-MYC antibody followed by immunoblot with an anti-FLAG to reveal interaction with FLAG-TRAF3 (top panel). Immunoprecipitated MAVS was revealed by immunoblot with anti-MYC antibody (second panel). Input for TRAF3 is shown (bottom panel). (F) IFNa4-Luc + IRF-7 promoter activity is shown for either empty vector, TRAF3 Y440A/Q442A, MAVS wt or in combination as indicated. Luciferase activity was analyzed at $24 \mathrm{~h}$ post transfection as per manufacturer's recommendation (Promega) and fold activation was determined compared to empty vector; values represent the average \pm S.D. Results are representative of at least three experiments run in triplicate. 
mediated IFN induction.

Reconstitution of the MAVS ${ }^{--}$MEFs with MAVS-Cterm $A A A$ does not restore IFN response

The next approach to investigate functional consequences of MAVS-TRAF3 interaction was to reconstitute $\mathrm{MAVS}^{-/-}$MEFs, which are unable to mount an antiviral response against RNA virus infection [62], with empty control vector, MAVS wt or MAVS-Cterm AAA expression plasmid and determine the magnitude of the antiviral response. Reconstitution with MAVS wt induced endogenous IFNBI, IFNA4, IFIT1, IL6 and IP10 mRNA, whereas reconstitution with MAVS Cterm AAA and subsequent vesicular stomatitis virus (VSV) infection reduced ISG expression by $50-80 \%$ (Figure $4 \mathrm{~A})$. These results were confirmed by monitoring the activity of IFN $\alpha 4$, IFN $\beta$, ISG56 and NF- $\kappa$ B promoters in reconstituted $\mathrm{MAVS}^{-/-}$MEFs; MAVS-Cterm AAA was a poor inducer of all promoters, compared with MAVS wt (Figure 4B).

Next, reconstituted MAVS ${ }^{-/-}$MEFs were infected with GFP-expressing VSV (designated as GFP-VSV- $\Delta 51$ ) [63, 64] at a MOI of five and virus replication was directly monitored by flow cytometry. MEFs reconstituted with empty vector were $>80 \%$ GFP positive and thus highly permissive for virus replication, whereas cells reconstituted with MAVS wt expressed very little GFP (Figure 4C). In $\mathrm{MAVS}^{-/-}$MEFs reconstituted with MAVSCterm AAA, the cells were also highly permissive for VSV with $>60 \%$ of cells being GFP positive (Figure $4 C$ ), indicating that MAVS-Cterm AAA was unable to restore an antiviral state that blocked VSV replication. These results were confirmed by VSV plaque assays with the supernatant from VSV-infected MEFs (Figure 4C). MEFs reconstituted with MAVS wt did not produce any plaques, whereas cells reconstituted with empty vector or MAVS-Cterm AAA produced approximately $3 \times 10^{5}$ p.f.u./ml of VSV (Figure 4D), thus further illustrating the importance of the MAVS C-terminal TRAF3-binding site in generating an IFN-responsive antiviral state.

\section{IKKE recruitment to MAVS influences TRAF3 binding to MAVS}

Because the C-terminal TIM is located adjacent to the IKK $\varepsilon$ interaction site, we were interested to determine if IKK $\varepsilon$ cooperatively influenced TRAF3 association with MAVS. MAVS wt and IKKe interaction was readily detected by co-immunoprecipitation (Figure 5A, lane 2), while IKK $\varepsilon$ and MAVS-Cterm AAA co-expression actually resulted in a modest increase in MAVS-IKK $\varepsilon$ interaction (Figure 5A, lane 3), suggesting that mutation of the C-terminal TIM facilitated IKK $\varepsilon$-MAVS complex formation. To follow this observation, the interaction between a constant amount of MAVS and TRAF3 was examined in the presence of increasing amounts of IKKe. A dose-dependent reduction in the binding between MAVS and TRAF3 was observed with increasing IKKe, suggesting that IKKe binding to MAVS may interfere with TRAF3 binding to MAVS (Figure 5B, lanes 3-7). Furthermore, similar results were obtained when the kinase dead mutant of IKK $\varepsilon$ (IKK $\varepsilon$ K38A) was used in the experiment, indicating that the kinase activity of IKK $\varepsilon$ was not necessary to dislodge TRAF3 from MAVS (Figure $5 \mathrm{C}$, lanes 3-7). Note, however, that the slower migration of MAVS in the presence of IKK $\varepsilon$ (Figure 5B, lanes 3-7), but not in the presence of IKKeK38A (Figure 5C, lanes 3-7), indicates that MAVS is a target of IKK $\varepsilon$-mediated phosphorylation, as previously reported [15].

To confirm that the displacement of TRAF3 occurred via the C-terminus of MAVS, the interaction between a constant amount of C-terminal MAVS construct aa 364540 and TRAF3 was examined in the presence of increasing amounts of IKK $\varepsilon$; a dose-dependent reduction in the binding between MAVS aa 364-540 and TRAF3 was observed with increasing IKKe (Figure 5D, lanes 4-7), indicating that IKK $\varepsilon$ binding to MAVS disrupted TRAF3 binding to the TIM domain located in the C-terminus of MAVS. Of note, the level of TRAF3 protein was also reduced with increasing amounts IKK $\varepsilon$ or IKK\&K38A (Figure 5B-5D, input TRAF3), suggesting a role for the IKKE-MAVS complex in TRAF3 protein turnover.

IKKE-mediated disruption of the MAVS-TRAF3 complex requires Lys500

As demonstrated previously, mutation of MAVS Lys500 to Arg500 abrogated IKK $\varepsilon$ recruitment to MAVS [15]; therefore, we next compared MAVS(K500R)TRAF3 association in the presence or absence of IKKE. As expected, a fivefold decrease in TRAF3-MAVS binding was observed when IKK $\varepsilon$ was co-expressed, as compared with MAVS-TRAF3 interaction in the absence of IKK $\varepsilon$ (Figure 6A, lanes 1, 2). Strikingly, the association between TRAF3-MAVS(K500R) was not affected by increasing amounts of IKKe (Figure 6A, lanes 3, 4 ), indicating that in the absence of IKK $\varepsilon$ binding to MAVS(K500R), the MAVS-TRAF3 interaction was not disrupted. This result implies that the recruitment of IKK $\varepsilon$ to MAVS may disrupt the tethering of the TRAF3containing IFN signaling complex to the TIM domain.

To address the issue of TRAF3 turnover, total protein immunoblots were performed in cells co-expressing TRAF3 and increasing amounts of IKK $\varepsilon$ or IKK $\varepsilon$ K38A in the presence of either MAVS wt or MAVS(K500R) (Figure 6B-6E). A dose-dependent decrease in TRAF3 
A

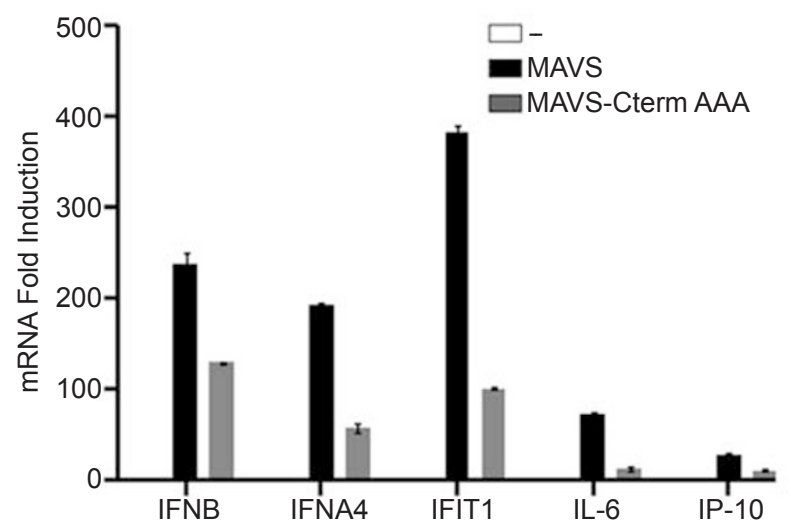

C

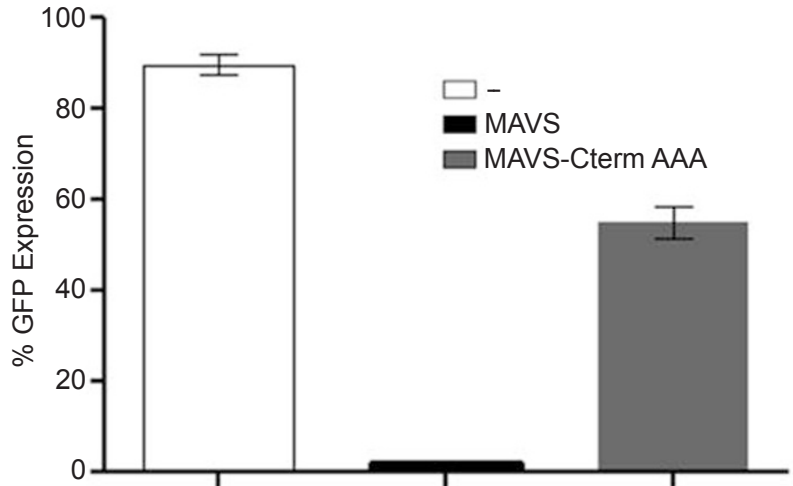

B

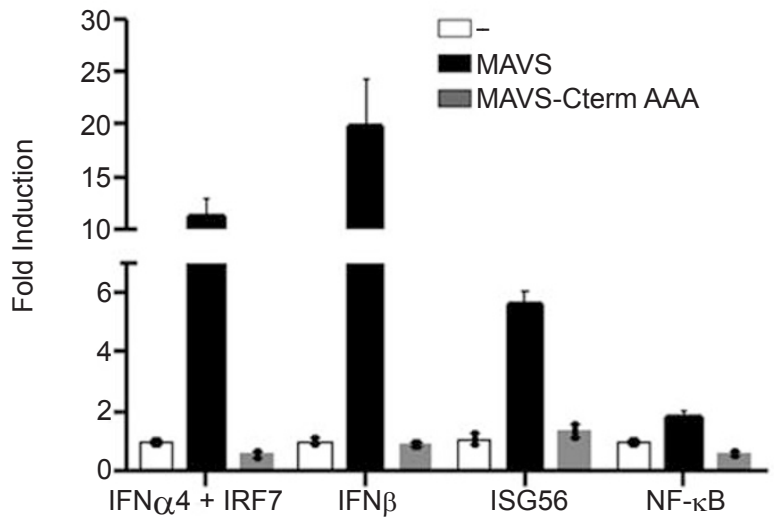

D

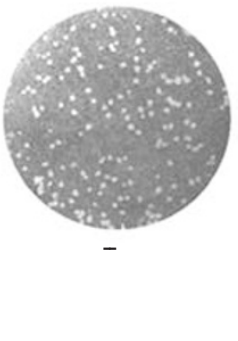

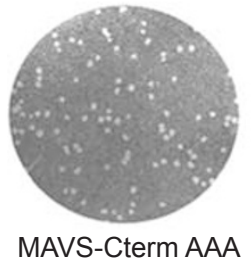

Figure 4 Reconstitution of MAVS ${ }^{-/}$MEFs with MAVS-Cterm AAA does not lead to a proper antiviral response. (A) qPCR analysis of total RNA isolated from $\mathrm{MAVS}^{-/-}$MEF cells reconstituted with either: empty vector, MAVS wt or MAVS-Cterm AAA. Relative fold expression levels of IFNB, IFNA4, IFIT1 (ISG56), IL6 and IP-10 versus ACTIN mRNA are shown. Data is representative of at least two experiments run in duplicate. (B) MAVS ${ }^{-/}$MEF cells were reconstituted with IFN $\alpha 4-L u c$ (IRF7), IFN $\beta$ Luc, ISG56-Luc or NF-kB reporter plasmid and expression plasmids encoding either empty, MAVS wt or MAVS-Cterm AAA as indicated. Luciferase activity was analyzed at $24 \mathrm{~h}$ post transfection and fold activation was determined compared with empty vector; values represent the average \pm S.D. Results are representative of at least three experiments run in triplicate. (C) $\mathrm{MAVS}^{-/-}$MEFs were reconstituted with either: empty vector, MAVS wt or MAVS-Cterm AAA for $24 \mathrm{~h}$ followed by VSV- $\triangle 51-$ GFP infection at an m.o.i. of 5. Samples were collected at $48 \mathrm{~h}$ post infection. VSV replication was determined by flow cytometry for GFP expression. Results are shown as an average GFP percentage for two independent experiments. (D) Virus titers determined by standard plaque assay using supernatant from (C). Images of the assay plate are also shown.

levels was detected with increasing amounts of IKK $\varepsilon$ expression (Figure 6B); however, no change in TRAF3 protein level was observed in the presence of MAVS(K500R) (Figure 6C), thus demonstrating that IKKe recruitment to MAVS contributes to the turnover of TRAF3 protein. Furthermore, IKKE kinase activity was not required for TRAF3 turnover, since increasing the amounts of IKK $\varepsilon$ K38A also diminished TRAF3 levels (Figure 6D). To reinforce that IKK $\varepsilon$ binding was required for TRAF3 turnover, IKKeK38A was also expressed in the presence of MAVS(K500R); without IKKeK38A binding to MAVS, the level of TRAF3 remained constant (Figure $6 \mathrm{E})$. Altogether, these experiments argue that the forma- tion of a MAVS-IKKe-TRAF3 tripartite complex leads to a disruption in TRAF3 binding and increased turnover of TRAF3 protein, as previously described [62].

\section{Identification of the MAVS-IKKE-TRAF3 complex in} Sendai-virus-infected cells

To confirm the spatiotemporal events described above in a physiologically relevant context, a kinetic analysis of the MAVS-IKKE-TRAF3 complex was performed in A549 cells infected with $\mathrm{SeV}$ (50 HAU/ml; Figure 7). Following immunoprecipitation of endogenous MAVS, RIG-I was detected in the immunoprecipitate as early as $4 \mathrm{~h}$ post infection and RIG-I association with MAVS in- 

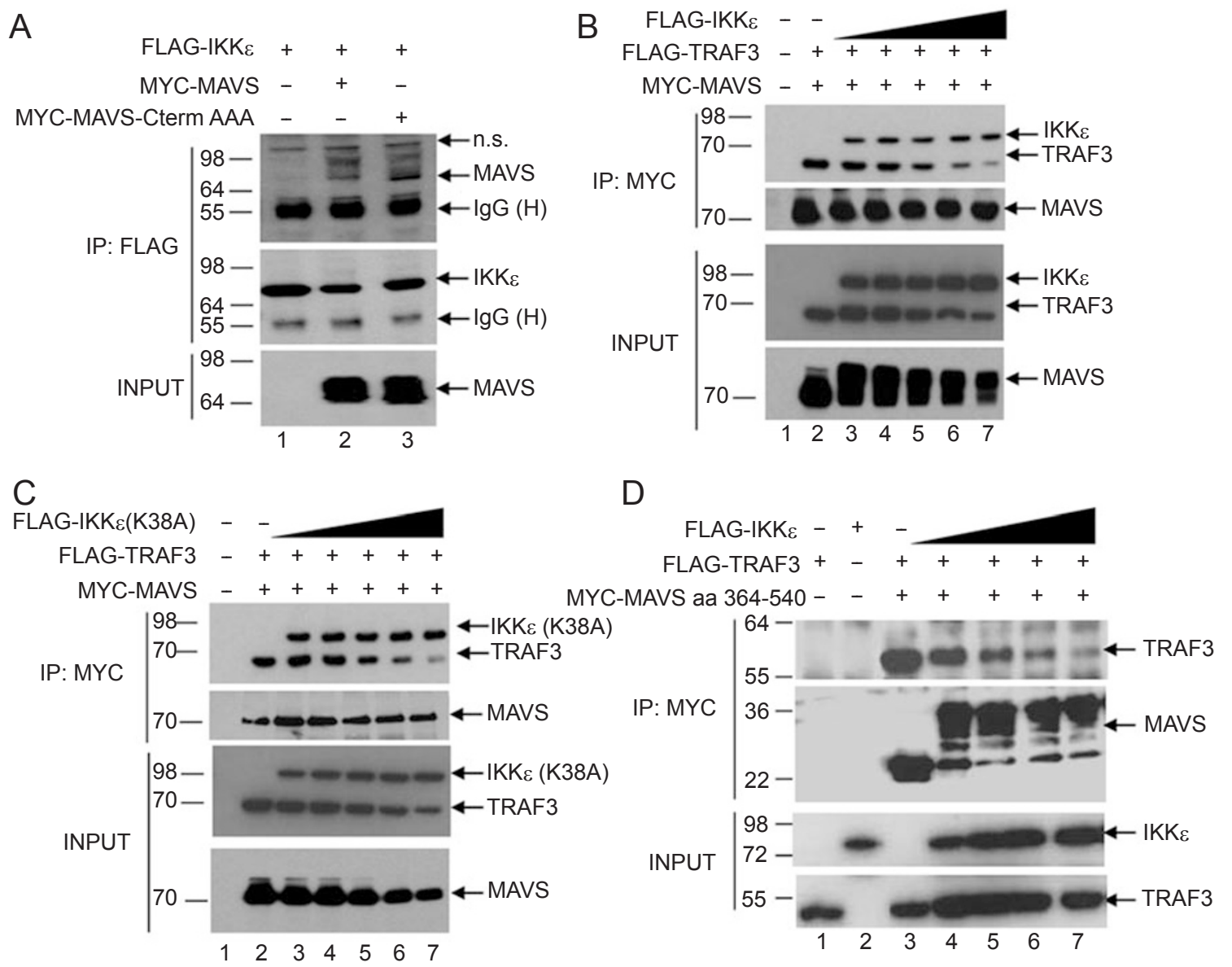

Figure 5 IKKE influences TRAF3-binding to the C-terminal TIM of MAVS. (A) HEK293 cells were transfected with either: empty vector, FLAG-IKK $\varepsilon$ or in combination with either MYC-MAVS or MYC-MAVS-Cterm AAA. Interaction between MAVS wt and MAVS-Cterm AAA with IKKE was revealed using an anti-MYC antibody following immunoprecipitation with anti-FLAG antibody (upper panel). Immunoprecipitated IKKE was determined using and anti-FLAG antibody (second panel). Input of transfected MAVS is also shown using an anti-MYC antibody (bottom panel). (B-D) Where indicated, FLAG-TRAF3 and MYC-MAVS (A, B), MYC-MAVS aa 364-540 (D) were transfected at the same amount either alone or in combination with FLAG-IKKE (B, D) or FLAG-IKK\&K38A (C) in increasing amounts. TRAF3 and IKK 8 or IKK\&K38A interaction were uncovered using an anti-FLAG antibody. Immunoprecipitated MAVS wt (B, C) or MAVS aa 364-540 (D) using an anti-MYC antibody is shown. Input for IKK $\varepsilon$ and TRAF3 and MAVS wt are shown using an anti-FLAG or anti-MYC antibody.

creased to a constant level between 6-24 h. Concomitant with the MAVS-RIG-I interaction, TRAF3 binding was detected, including a TRAF3 band at $\sim 100 \mathrm{kDa}$, corresponding to a K63-linked ubiquitinated form of TRAF3, as previously described [59] (Figure 7). IKK $\varepsilon$ was also detected in the immunoprecipitate at 4-8 h (Figure 7, lanes 3-5) [15]. Concomitant with IKK $\varepsilon$ dissociation from MAVS at $10 \mathrm{~h}$ was the disappearance of the $\sim 100$ kDa TRAF3 band (Figure 7, lane 6). As a measure of IFN signaling activity, the formation of the RIG-I-MAVS-TRAF3 interactome resulted in IRF3 phosphorylation at 4-12 h, as detected by the Ser396 phospho-specific Ab $[40,65]$. As TRAF3 binding to MAVS decreased, both TRAF3 and IRF3 protein levels also decreased, thus, confirming previous observations that TRAF3 and IRF3 undergo proteasomal degradation following virus infection (Figure 7, lane 8) [66, 67], both of which are hallmarks of the termination of the IFN response.

\section{Discussion}

This study characterizes a novel functional TRAF3binding site located at the C-terminus of MAVS - the TRAF-interacting motif 455-PEENEY-460. Several lines of evidence support the importance of this site in mediating TRAF3-dependent IFN signaling: (1) binding of 


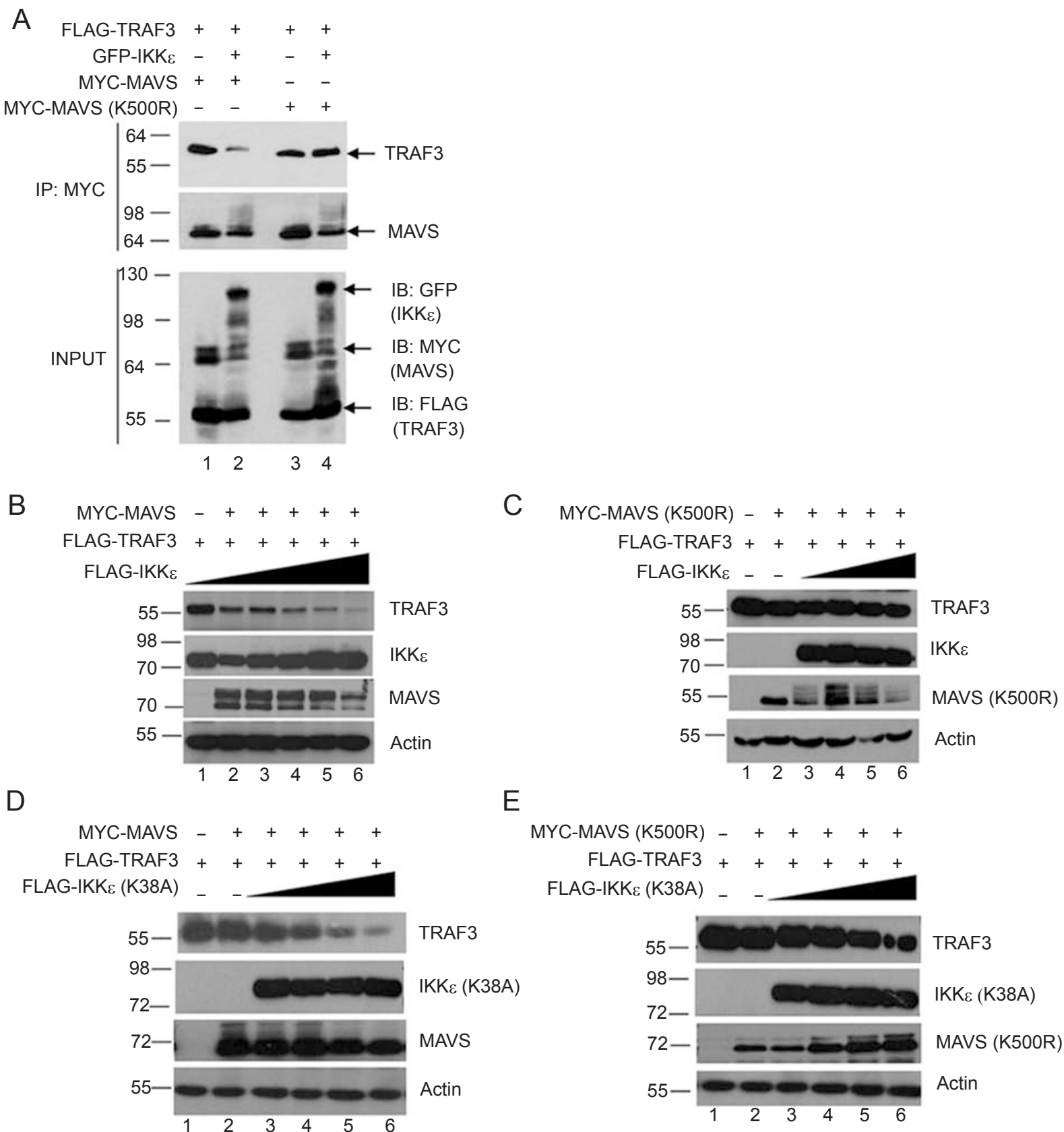

Figure 6 Recruitment of IKK to MAVS increases TRAF3 turnover. (A) HEK293 cells were transfected with the following expression plasmids: FLAG-TRAF3, MYC-MAVS or MYC-MAVS(K500R). Where indicated, GFP-IKKE was also co-transfected. Interaction between MAVS and TRAF3 was observed following immunoprecipitation of MAVS using an anti-MYC antibody and immunoblot for TRAF3 using a FLAG antibody (upper panel). Equal amounts of MAVS and its mutant K500R is demonstrated using an anti-MYC antibody following immunoprecipitation with anti-MYC (second panel). Inputs for IKKE (anti-GFP), MAVS (anti-MYC) and TRAF3 (anti-FLAG) are also shown (bottom panel). (B-E) HEK293 cells were transfected with either: (B) equal amounts of FLAG-TRAF3, MYC-MAVS or increasing amounts of FLAG-IKKE; (C) MYC-MAVS(K500R) was transfected with equal amounts of FLAG-TRAF3 with increasing amounts of FLAG-IKKE; (D) equal amounts of FLAG-TRAF3, MYCMAVS or increasing amounts of FLAG-IKKe(K38A); (E) MYC-MAVS(K500R) was transfected with equal amounts of FLAGTRAF3 with increasing amounts of FLAG-IKK $\varepsilon(\mathrm{K} 38 \mathrm{~A})$. Western blot analysis was used to detect the protein levels of TRAF3, IKKE and IKK $\varepsilon(\mathrm{K} 38 \mathrm{~A})$ using an anti-FLAG antibody, and detection of MAVS and MAVS(K500R) was revealed using an antiMYC antibody. Actin antibody was used as a loading control. 


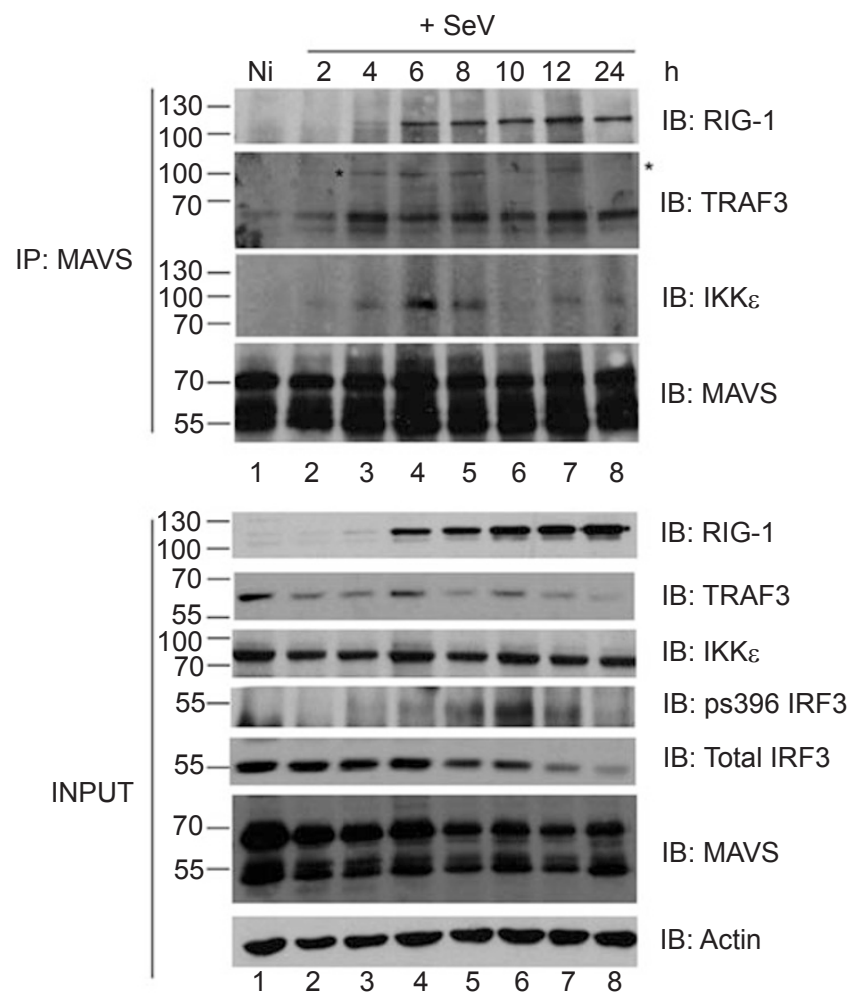

Figure 7 Endogenous tripartite interaction amongst MAVS, TRAF3 and IKKE. Lung carcinoma cell line A549 were infected with $\mathrm{SeV}(50 \mathrm{HAU} / \mathrm{ml})$ for the indicated times. Endogenous MAVS was immunoprecipitated using an anti-MAVS antibody and interaction with RIG-I, TRAF3 and IKKE were revealed using an anti-RIG-I (top panel), anti-TRAF3 (second panel), antiIKK $\varepsilon$ antibody (third panel). Equal amounts of immunoprecipitated MAVS was revealed using an anti-MAVS antibody (fourth panel). Input for RIG-1, TRAF3, IKKE, IRF3 (ps396 IRF3 and TOTAL IRF3), MAVS and Actin are shown (last seven panels).

TRAF3 was abrogated using MAVS-Cterm AAA mutant (455-AAANEY-460), and TRAF3 binding to the C-terminal TIM was essential for the IFN antiviral response; (2) mutation of the previously characterized N-terminal TIM (143-PVQET-147) did not affect TRAF3 binding or downstream IFN signaling; (3) a non-binding mutant of TRAF3, mutated at amino acids Y440 and Q442 did not interact with the TIM or induce IFN signaling; (4) MAVS-Cterm AAA was unable to reconstitute an antiviral state in $\mathrm{MAVS}^{-/-}$MEFs; and (5) TRAF3 was displaced from MAVS when IKK $\varepsilon$ was present, a mechanism independent of its kinase activity but dependent on K63 linked ubiquitination of MAVS at Lys500. Interestingly, the stability of TRAF3 was minimally affected when MAVS 364-540 and IKK $\varepsilon$ were present in our system, although the displacement of TRAF3 from MAVS was readily detected with increasing IKKE. MAVS protein contains multiple regulatory domains and it is likely that the N-terminus of MAVS may also contribute to the stability of TRAF3. These results thus highlight the interplay of C-terminal binding of TRAF3 and IKK $\varepsilon$ in the shutdown of the IFN response.

In the context of several recent studies, the current observations support a multistep model of regulation of the IFN antiviral response, mediated via TRAF3 interactions with the MAVS scaffold (Figure 8). Sensing of viral RNA structures by RIG-I results in Lys63 ubiquitination of RIG-I by TRIM25, leading to a conformational change in RIG-I that facilitates CARD-CARD domain interactions with MAVS [68] (reviewed in [26]). MAVS dimerizes [15-17] and recruits TRAF3 as an essential step in the activation of the antiviral response (Figure $8 \mathrm{~A})[12,18,35]$. This study demonstrates the critical role of the C-terminal 455-PEENEY-460 TIM in recruiting TRAF3; and the recruitment requires Lys63-polyubquitination of TRAF3 by an unidentified E3 ubiquitin ligase, or possibly by auto-ubiquitination $[26,59]$. The interaction of the TRAF3-containing signaling complex (Figure 8, panel A) activates downstream kinases TBK1/IKK $\varepsilon$ that phosphorylate $\mathrm{C}$-terminal serine residues of latent cytoplasmic IRF3 and IRF7 [40-42]. IRF3 and/or IRF7 then dimerize, translocate into the nucleus and bind to specific IRF-response elements in the IFN $\beta$ promoter, as well as other type I IFN genes [69]. Subsequently, Lys63 polyubiquitination of MAVS at Lys500 leads to the recruitment of IKK $\varepsilon$ to the C-terminus of MAVS [15], and initiates a termination of the IFN response, by disrupting TRAF3 binding to MAVS (Figure 8, panel B). Kayagaki et al. [31] discovered, through small interfering RNA screen, that the DUBA specifically removed Lys63linked polyubiquitin chains from TRAF3, resulting in its dissociation from the IFN signaling complex, contributing to the negative regulation of type I IFN response (Figure 8, panel C). De-ubiquitinated TRAF3 is then targeted for Lys48 ubiquitination mediated by the E3 ubiquitin ligase TRIAD3A [59]; finally TRAF3 is degraded by a proteasome-dependent mechanism, thus removing an essential adapter involved in the tethering of the IFN signaling complex to MAVS (Figure 8, panel C). TRAF3 undergoes biphasic ubiquitination following viral infection: early after infection, Lys63-linked polyubiquitination contributes to IFN signaling complex formation, while at later times after infection, TRAF3 undergoes Lys48-linked polyubiquitination mediated by TRIAD3A that targets TRAF3 for proteasomal degradation (Figure 8, panel C) [59].

The complexity of the regulatory interactions at the MAVS scaffold is highlighted further by the identification of several other proteins that interact with MAVS, 


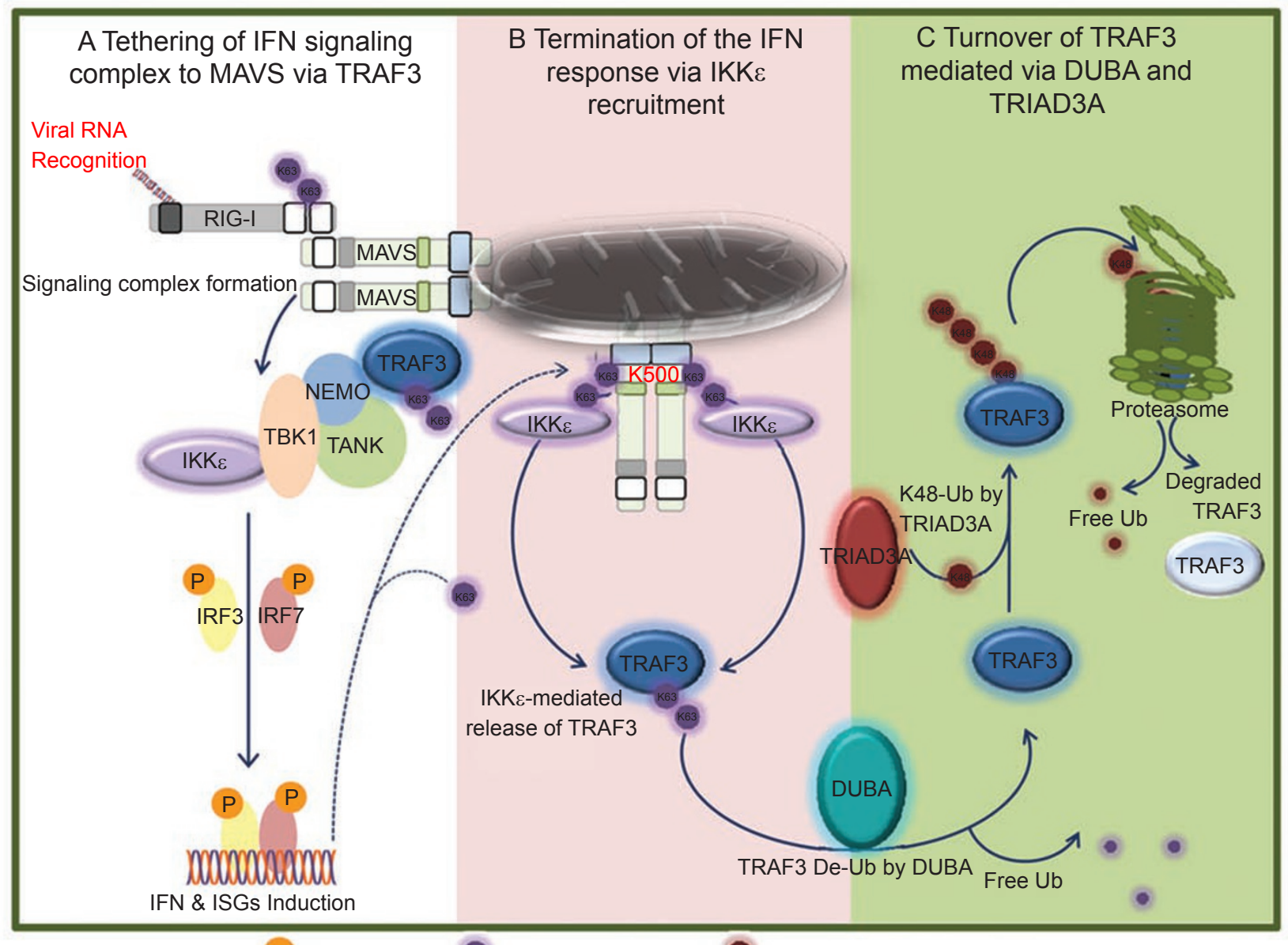

P $=$ Phosphorylation

$=$ K63-linked Ubiquitination

Figure 8 Schematic representation of TRAF3-mediated positive and negative regulation of IFN signaling. Sensing of dsRNA and Lys63 ubiquitination of RIG-I by TRIM25 leads to a conformational change that allows the CARD domain to interact with downstream adaptor MAVS. MAVS in turn dimerizes and recruits TRAF3 to the C-terminal 455-PEENEY-460 consensus site, a process requiring Lys63-polyubquitination of TRAF3. The MAVS-TRAF3 interaction leads to the recruitment and activation of downstream kinases TBK1/IKKE to phosphorylate IRF3 and IRF7 in the cytoplasm. IRF3 and IRF7 will dimerize, translocate into the nucleus where they will bind specific ISGs and induce type I IFN. K63-linked polyubiquitination at Lys500 of MAVS recruits IKK $\varepsilon$ to the C-terminus of MAVS. The recruitment of IKKE to the mitochondria will release TRAF3 from the mitochondria and initiate the signal to shutdown the IFN response by either activating or recruiting DUBA to remove the Lys63polyubiquitin chains on TRAF3. De-ubiquitinated TRAF3 is then targeted to the proteasome by Lys48 ubiquitination mediated by the action of TRIAD3A and thus shutting down any further activation of the IFN pathway.

including: TRAF2/5/6, caspase-8/10, RIP1, FADD, TRADD, MITA/STING/MYPS/ERIS, RNF125, REUL, A20, CYLD, Atg5-Atg12, NLRX1, NLRC5, LGP2 (reviewed in [19]), proteins that may further contribute to positive or negative regulation of the RIG-I/MAVS pathway. To further add to the complexity of MAVS regulation of signaling, Dixit et al. [70] recently demonstrated that MAVS can localize to cytosolic peroxisome structures, which are formed from the endoplasmic reticulum and are usually involved in cellular metabolism [71]. MAVS localization to the peroxisome establishes a dis- tinct population of MAVS involved in the early, transient response against viral pathogens, while mitochondrial MAVS produces a sustained robust antiviral response. Interestingly, peroxisomal MAVS and mitochondrial MAVS may not utilize the same transcription factors to induce antiviral genes. It will be interesting to determine if the adapters tethered to peroxisomal MAVS differ from those associated with mitochondrial MAVS. Recently, Zeng et al. [72] established a cell-free system that will permit further dissection of the pathway and contribute to a better understanding of events leading to the host 
antiviral and inflammatory responses. In conclusion, we provide evidence that the $\mathrm{C}$-terminal regulatory region of MAVS is involved in positive and negative regulation of the antiviral response through regulatory interactions with TRAF3 and IKKe.

\section{Materials and Methods}

\section{Plasmid construction and mutagenesis}

Plasmids encoding FLAG-IKKe, FLAG-IKK\&K38A, GFPIKK $\varepsilon$, FLAG-TRAF3, MYC-TRAF3, MYC-MAVS, MYC-MAVS (aa $1-155$, aa $1-400$, aa $1-580$, aa $157-400$, aa $151-540$, aa $364-$ 540, aa 468-540 and aa 503-540), MYC-MAVS(K500R), FLAGMAVS $\Delta$ aa 101-450, FLAG-MAVS $\triangle$ aa 101-480 have been previously described [14-15, 29, 40,59]. MAVS multisite point mutants at proline $455(\mathrm{P})$, glutamic acid $456(\mathrm{E})$, glutamic acid $457(\mathrm{E})$ to alanine (A) (MYC-MAVS-Cterm AAA) and proline 143, glutamine 145, threonine 147 to alanine (MYC-MAVS-Nterm AAA), were generated by multisite directed mutagenesis as per manufacturer's instructions (Stratagene, La Jolla, CA, USA). All point mutants were verified by sequencing.

\section{Tissue culture, transfection techniques and virus infection}

HEK293, Vero and HeLa cells were grown in DMEM supplemented with 10\% FBS (Wisent, St-Bruno, Quebec, Canada). A549 cells were cultured in F12K media supplemented with 10\% FBS (Wisent). MAVS ${ }^{--}$MEFS (kind gift from Dr Zhijian Chen) and TRAF3 $^{-/-}$MEFs (kind gift from Dr Genhong Cheng) were cultured DMEM 10\% FBS (Invitrogen, Carlsbad, CA, USA), supplemented with $1 \%$ non-essential amino acids and $1 \%$ L-glutamine. Culture media and supplements were purchased from Wisent. Where indicated, A549 cells were infected with Sendai virus at $50 \mathrm{HAU} /$ $\mathrm{ml}$ (Charles River Lab) in serum free condition supplemented with serum $1 \mathrm{~h}$ post infection. Transient transfections were carried out in subconfluent HEK293 cells by calcium phosphate, by Fugene6 (Roche Diagnostics, Mannheim, Germany), in HeLa cells or Lipofectamine 2000 in knockout MEFs (Invitrogen) when indicated, as per manufacturer's recommendations.

\section{VSV infection and viral replication}

Where indicated MAVS ${ }^{-/}$cells were infected with 5 m.o.i of VSV (strain $\Delta 51$; kind gift from Dr John Bell) in serum free condition supplemented with serum $1 \mathrm{~h}$ post infection. Flow cytometry (1 $\times 10^{4}$ cells per measurement) was performed with a FACS Calibur (Becton-Dickinson, Mississauga, Ontario, Canada) and analyzed with Cell Quest software and FCS Express version 3 (De Novo Software, Los Angeles, CA) for GFP expression. Plaque assay was done using confluent monolayers of Vero cells in six-well plates were infected with $0.1 \mathrm{ml}$ of serially diluted samples; after $1 \mathrm{~h}$ of infection, at $37{ }^{\circ} \mathrm{C}$, medium was removed and replaced with complete medium containing $0.5 \%$ methyl cellulose (Sigma-Aldrich, Oakville, Ontario, Canada) for $48 \mathrm{~h}$. Vero cells were fixed in $4 \%$ formaldehyde and stained with crystal violet.

\section{Reporter gene assay}

Subconfluent HeLa or confluent MAVS ${ }^{-/}$MEFs cells were transfected with $20 \mathrm{ng}$ of pRLnull reporter (Renilla luciferase, in- ternal control), $100 \mathrm{ng}$ of pGL3-IFN $\alpha 4$, pGL3-IFN $\beta$, pGL3-ISG56, pGL3-NF- $\kappa$ B, and $100 \mathrm{ng}$ of MYC-MAVS (wt or its mutated or truncated forms) as indicated. As previously described, IFN $\alpha 4$ promoter activity was measured in the presence of exogenous IRF7 at $100 \mathrm{ng}$ [40]. At $24 \mathrm{~h}$ post transfection, reporter gene activity was measured by Dual-Luciferase reporter assay according to the manufacturer's instructions using GLIOMAX 20/20 luminometer (Promega Corporation, Madison, WI, USA). Three independent experiments were carried out in triplicate. Error bars represent the mean standard deviation for triplicates and analyzed using Prism 5 software.

\section{Western blot and co-immunoprecipitation}

Whole-cell extracts (WCE, 30-50 $\mu \mathrm{g}$ ) were separated in 7.5 or $10 \%$ acrylamide gel by SDS-PAGE and transferred to a nitrocellulose membrane (BioRad, Mississauga, ON, Canada). Membranes were blocked for $1 \mathrm{~h}$ at room temperature in $5 \%(\mathrm{v} / \mathrm{v})$ dried milk/0.1\% (v/v) Tween-20 in PBS and then were probed with primary antibodies: for $1 \mathrm{~h}$ at room temperature or overnight at $4{ }^{\circ} \mathrm{C}$. After washes, membranes were incubated with horse-radish peroxidase-coupled secondary antibody solutions (1:3000 in 5\% milk/ PBS, KPL, Gaithersburg, MD, USA) for $1 \mathrm{~h}$ at room temperature, washed and revealed using ECL reagent (Perkin-Elmer, Waltham, MA, USA) according to manufacturer's instructions.

HEK293 cells were transiently transfected with the indicated expression plasmids. Cells were harvested and immediately lysed in a $1 \%$ Triton $\mathrm{X}-100$ lysis buffer $(20 \mathrm{mM}$ Tris- $\mathrm{HCl}, \mathrm{pH}$ $7.5,150 \mathrm{mM} \mathrm{NaCl}, 1 \%$ Triton X-100, 10\% glycerol, $40 \mathrm{mM}$ $\beta$-glycerophosphate, $0.1 \%$ protease inhibitor cocktail, $1 \mathrm{mM}$ PMSF, $1 \mathrm{mM} \mathrm{Na} \mathrm{VO}_{4}, 5 \mathrm{mM} \mathrm{NaF}, 1 \mathrm{mM}$ DTT, $10 \mathrm{mM} \mathrm{NEM}$ ). Immunoprecipitation was carried out in WCE $(500 \mu \mathrm{g})$ with $1 \mu \mathrm{g}$ of anti-MYC (9E10; Santa Cruz Biotechnology, Santa Cruz, CA, USA) or anti-FLAG (Sigma-Aldrich, St Louis, MO, USA) coupled to $50 \mu 1$ of A/G PLUS-Agarose beads (Santa Cruz Biotechno$\operatorname{logy}$ ) at $4{ }^{\circ} \mathrm{C}$ with constant agitation. Following five washes with supplemented lysis buffer, samples were denatured in $2 \%$ SDSloading dye, separated by SDS-PAGE and transferred to a nitrocellulose membrane (BioRad). Co-immunoprecipitated TRAF3 or IKK $\varepsilon$ was detected by anti-FLAG antibody (Sigma-Aldrich) or by anti-GFP antibody (Roche Diagnostics).

For endogenous protein interactions, A549 cells were infected or not with $\mathrm{SeV}$ as described. At 2, 4, 6 and 8, 10, 12 and $24 \mathrm{~h}$ post-infection cells were harvested and lysed as above. Immunoprecipitation was carried out on WCE (1 mg) using an anti-MAVS antibody raised against the C-terminal (rabbit polyclonal, in collaboration with Millipore Corporation Cat: 06-1044; Temecula, $\mathrm{CA}, \mathrm{USA}$ ) for $16 \mathrm{~h}$ at $4{ }^{\circ} \mathrm{C}$ with constant agitation. Following washes, samples were analyzed by immunoblot. Co-immunoprecipitated RIG-I was detected using an anti-RIG-I antibody raised against the N-terminal (rabbit polyclonal, in collaboration with Millipore Corporation Cat: 06-1040; Temecula, CA, USA) TRAF3 (Santa-Cruz, G-6), IKK $\varepsilon$ was detected using an anti-IKK $\varepsilon$ mouse monoclonal antibody (in collaboration with BD Biosciences, San Jose, CA, USA). Other antibodies used in this study include IRF3 ps396 (Millipore Corporation; Temecula, CA, USA); anti-IRF3 antibody raised against the C-terminal (Rabbit polyclonal, in collaboration with Millipore Corporation Cat:06-1045; Temecula, CA, USA); anti-actin (Millipore Corporation; Temecula, CA, USA). 


\section{Quantitative real-time PCR}

DNase-treated total RNA from HeLa cells and MAVS ${ }^{-/}$cells transfected with MYC-MAVS wt, internal deletion constructs or mutated AAA was prepared using the RNeasy kit (Qiagen, Mississauga, ON, Canada). RNA concentration was determined by absorption at $260 \mathrm{~nm}$, and RNA quality was ensured by a $260 / 280$ ratio $\geq 2.0$. Total RNA was reverse transcribed with $100 \mathrm{U}$ of Superscript II Plus RNAse H reverse transcriptase using oligo AnCT primers (Gibco BRL Life Technologies, CA, USA). qPCR assays were performed using the SYBR Green I on a Light Cycler apparatus (Roche Diagnostics). Human primers sequences used in this study are as follows: IFNBI forward: 5'-TTGTGCTTCTCCACTACAGC-3', IFNBI reverse: 5'-ATCTGAGGTGCCCATGCTAC-3'; IFNA2 forward: 5'-CCTGATGAAGGAGGACTCCAT-3', IFNA2 reverse: 5'-AAAAAGGTGAGCTGGCATACG-3'; IFIT1 forward: 5'-CAACCAAGCAAATGTGAGGA-3', IFIT1 reverse: 5'-AGGGGAAGCAAAGAAAATGG-3'; IL-6 forward: 5'-GGAGACTTGCCTGGTGAAAA-3', IL-6 reverse: 5'-ATCTGAGGTGCCCATGCTAC; $I P-10$ forward: 5 '-TCTTCTCACCCTTCTTTTTCATTGT-3', IP-10 reverse: 5'-TTCCTGCAAGCCAATTTTGTC-3'; OASl forward: 5'-CCTGGTTGTCTTCCTCAGTCC-3', OASI reverse: 5'-GTGGAGAACTCGCCCTCTTT-3'; RIG-I forward: 5'-GCAGAGGCCGGCATGAC-3', RIG-I reverse: 5'-TGTAGGTAGGGTCCAGGGTCTTC-3'; ACTIN forward: 5'-ACTGGGACGACATGGAGAAAA-3', ACTIN reverse: 5'-GCCACACGCAGCTC-3'. Murine primers sequences used in this study are as follows: IFNB forward: 5'-CACAGCCCTCTCCATCAACT-3', IFNB reverse: 5'-TCCCACGTCAATCTTTCCTC-3'; IFNA4 forward: 5'-GAAGGACAGGAAGGATTTTGGA-3', IFNA4 reverse: 5'-TGAGCCTTCTGGBATCTGTTGGT-3'; IFIT 1 forward: 5'-CTCTGAAAGTGGAGCCAGAAAAC-3', IFIT1 reverse: 5'-AAATCTTGGCGATAGGCTACGA-3'; $I P-10$ forward: 5'-AAGTGCTGCCGTCATTTTCT-3', $I P-10$ reverse: 5'-CACTGGGTAAAGGGGAGTGA-3'; $A C T I N$ forward: 5'-CACCAGTTCGCCATGGAT-3', ACTIN reverse: 5'-CCTCGTCACCCACATAGGAG- $3^{\prime}$. Murine $I L-6$ primers were obtained from Origene (catalog number: MP206798). PCR efficiency results were obtained from duplicate measurements of two individual cDNA samples. Experiments were performed at least twice. All data are presented as a relative quantification, based on the relative expression of target genes versus $A C T I N$ as reference gene and analyzed using Prism 5 software.

\section{Acknowledgments}

This work was supported by grants from the Canadian Institutes for Health Research, Canadian Cancer Society and the Canadian Foundation for AIDS Research, awarded to JH and grants from L'Agence Nationale de la Recherche contre le SIDA (ANRS), awarded to EM. SP is the recipient of an FRSQ Bourse de Troisième Cycle (Doctorat) and MV was the recipient of a FRM Bourse de Fin de Thèse.

\section{References}

1 Samuel CE. Antiviral actions of interferons. Clin Microbiol Rev 2001; 14:778-809.

2 Yoneyama M, Fujita T. Recognition of viral nucleic acids in innate immunity. Rev Med Virol 2010; 20:4-22.
3 Akira S, Uematsu S, Takeuchi O. Pathogen recognition and innate immunity. Cell 2006; 124:783-801.

4 Uematsu S, Akira S. Toll-like receptors and innate immunity. J Mol Med 2006; 84:712-725.

5 Schindler C, Levy DE, Decker T. JAK-STAT signaling: from interferons to cytokines. J Biol Chem 2007; 282:2005920063.

6 Yoneyama M, Kikuchi M, Natsukawa T, et al. The RNA helicase RIG-I has an essential function in double-stranded RNAinduced innate antiviral responses. Nat Immunol 2004; 5:730737.

7 Yoneyama M, Fujita T. Function of RIG-I-like receptors in antiviral innate immunity. J Biol Chem 2007; 282:1531515318.

8 Wilkins C, Gale M Jr. Recognition of viruses by cytoplasmic sensors. Curr Opin Immunol 2010; 22:41-47.

9 Komuro A, Horvath CM. RNA- and virus-independent inhibition of antiviral signaling by RNA helicase LGP2. $J$ Virol 2006; 80:12332-12342.

10 Kawai T, Takahashi K, Sato S, et al. IPS-1, an adaptor triggering RIG-I- and Mda5-mediated type I interferon induction. Nat Immunol 2005; 6:981-988.

11 Meylan E, Curran J, Hofmann K, et al. Cardif is an adaptor protein in the RIG-I antiviral pathway and is targeted by hepatitis C virus. Nature 2005; 437:1167-1172.

12 Seth RB, Sun L, Ea CK, Chen ZJ. Identification and characterization of MAVS, a mitochondrial antiviral signaling protein that activates NF-kappaB and IRF 3. Cell 2005; 122:669-682.

$13 \mathrm{Xu}$ LG, Wang YY, Han KJ, Li LY, Zhai Z, Shu HB. VISA is an adapter protein required for virus-triggered IFN-beta signaling. Mol Cell 2005; 19:727-740.

14 Lin R, Lacoste J, Nakhaei P, et al. Dissociation of a MAVS/ IPS-1/VISA/Cardif-IKKepsilon molecular complex from the mitochondrial outer membrane by hepatitis $\mathrm{C}$ virus NS3-4A proteolytic cleavage. J Virol 2006; 80:6072-6083.

15 Paz S, Vilasco M, Arguello M, et al. Ubiquitin-regulated recruitment of IkappaB kinase epsilon to the MAVS interferon signaling adapter. Mol Cell Biol 2009; 29:3401-3412.

16 Baril M, Racine ME, Penin F, Lamarre D. MAVS dimer is a crucial signaling component of innate immunity and the target of hepatitis C virus NS3/4A protease. J Virol 2009; 83:12991311.

17 Tang ED, Wang CY. MAVS self-association mediates antiviral innate immune signaling. J Virol 2009; 83:3420-3428.

18 Saha SK, Pietras EM, He JQ, et al. Regulation of antiviral responses by a direct and specific interaction between TRAF3 and Cardif. EMBO J 2006; 25:3257-3263.

19 Lin R, Paz S, Hiscott J. Tom70 imports antiviral immunity to the mitochondria. Cell Res 2010; 20:971-973.

20 Michallet MC, Meylan E, Ermolaeva MA, et al. TRADD protein is an essential component of the RIG-like helicase antiviral pathway. Immunity 2008; 28:651-661.

21 Balachandran S, Thomas E, Barber GN. A FADD-dependent innate immune mechanism in mammalian cells. Nature 2004; 432:401-405.

22 Tang ED, Wang CY. TRAF5 is a downstream target of MAVS in antiviral innate immune signaling. PLoS One 2010; 5:e9172.

23 Ishikawa H, Barber GN. STING is an endoplasmic reticulum adaptor that facilitates innate immune signalling. Nature 
2008; 455:674-678.

24 Zhong B, Yang Y, Li S, et al. The adaptor protein MITA links virus-sensing receptors to IRF3 transcription factor activation. Immunity 2008; 29:538-550.

25 Sun W, Li Y, Chen L, et al. ERIS, an endoplasmic reticulum IFN stimulator, activates innate immune signaling through dimerization. Proc Natl Acad Sci USA 2009; 106:8653-8658.

26 Nakhaei P, Genin P, Civas A, Hiscott J. RIG-I-like receptors: sensing and responding to RNA virus infection. Semin Immunol 2009; 21:215-222.

27 Moore CB, Bergstralh DT, Duncan JA, et al. NLRX1 is a regulator of mitochondrial antiviral immunity. Nature 2008; 451:573-577.

28 You F, Sun H, Zhou X, et al. PCBP2 mediates degradation of the adaptor MAVS via the HECT ubiquitin ligase AIP4. Nat Immunol 2009; 10:1300-1308.

29 Vitour D, Dabo S, Ahmadi Pour M, et al. Polo-like kinase 1 (PLK1) regulates interferon (IFN) induction by MAVS. J Biol Chem 2009; 284:21797-21809.

30 Mankouri J, Fragkoudis R, Richards KH, et al. Optineurin negatively regulates the induction of IFNbeta in response to RNA virus infection. PLoS Pathog 2010; 6:e1000778.

31 Kayagaki N, Phung Q, Chan S, et al. DUBA: a deubiquitinase that regulates type I interferon production. Science 2007; 318:1628-1632.

32 Lin R, Yang L, Nakhaei P, et al. Negative regulation of the retinoic acid-inducible gene I-induced antiviral state by the ubiquitin-editing protein A20. J Biol Chem 2006; 281:20952103.

33 He JQ, Oganesyan G, Saha SK, Zarnegar B, Cheng G. TRAF3 and its biological function. Adv Exp Med Biol 2007; 597:48-59.

34 Dempsey PW, Doyle SE, He JQ, Cheng G. The signaling adaptors and pathways activated by TNF superfamily. Cytokine Growth Factor Rev 2003; 14:193-209.

35 Oganesyan G, Saha SK, Guo B, et al. Critical role of TRAF3 in the Toll-like receptor-dependent and -independent antiviral response. Nature 2006; 439:208-211.

36 Karin M. How NF-kappaB is activated: the role of the IkappaB kinase (IKK) complex. Oncogene 1999; 18:6867-6874.

37 Karin M, Ben-Neriah Y. Phosphorylation meets ubiquitination: The control of NF- $\kappa \mathrm{B}$ activity. Ann Rev Immunol 2000; 18:621-663.

38 Perkins ND. Post-translational modifications regulating the activity and function of the nuclear factor kappa B pathway. Oncogene 2006; 25:6717-6730.

39 Perkins ND. Integrating cell-signalling pathways with NFkappaB and IKK function. Nat Rev Mol Cell Biol 2007; 8:4962.

40 Sharma S, tenOever BR, Grandvaux N, Zhou GP, Lin R, Hiscott J. Triggering the interferon antiviral response through an IKK-related pathway. Science 2003; 300:1148-1151.

41 Paz S, Sun Q, Nakhaei P, et al. Induction of IRF-3 and IRF7 phosphorylation following activation of the RIG-I pathway. Cell Mol Biol (Noisy-le-grand) 2006; 52:17-28.

42 Fitzgerald KA, McWhirter SM, Faia KL, et al. IKKepsilon and TBK1 are essential components of the IRF3 signaling pathway. Nat Immunol 2003; 4:491-496.

43 Panne D, McWhirter SM, Maniatis T, Harrison SC. Interferon regulatory factor 3 is regulated by a dual phosphorylationdependent switch. J Biol Chem 2007; 282:22816-22822.

44 Saitoh T, Yamamoto M, Miyagishi M, et al. A20 is a negative regulator of IFN regulatory factor 3 signaling. J Immunol 2005; 174:1507-1512.

45 Wang YY, Li L, Han KJ, Zhai Z, Shu HB. A20 is a potent inhibitor of TLR3- and Sendai virus-induced activation of NFkappaB and ISRE and IFN-beta promoter. FEBS Lett 2004; 576:86-90.

$46 \mathrm{Xu} \mathrm{L}$, Xiao N, Liu F, Ren H, Gu J. Inhibition of RIG-I and MDA5-dependent antiviral response by $\mathrm{gC} 1 \mathrm{qR}$ at mitochondria. Proc Natl Acad Sci USA 2009; 106:1530-1535.

47 Sanada T, Takaesu G, Mashima R, Yoshida R, Kobayashi T, Yoshimura A. FLN29 deficiency reveals its negative regulatory role in the Toll-like receptor (TLR) and retinoic acid-inducible gene I (RIG-I)-like helicase signaling pathway. J Biol Chem 2008; 283:33858-33864.

48 Zhang M, Wu X, Lee AJ, et al. Regulation of IkappaB kinaserelated kinases and antiviral responses by tumor suppressor CYLD. J Biol Chem 2008; 283:18621-18626.

49 Friedman CS, O'Donnell MA, Legarda-Addison D, et al. The tumour suppressor CYLD is a negative regulator of RIG-Imediated antiviral response. EMBO Rep 2008; 9:930-936.

50 Jia Y, Song T, Wei C, et al. Negative regulation of MAVS-mediated innate immune response by PSMA7. J Immunol 2009; 183:4241-4248.

51 Hou J, Wang P, Lin L, et al. MicroRNA-146a feedback inhibits RIG-I-dependent Type I IFN production in macrophages by targeting TRAF6, IRAK1, and IRAK2. J Immunol 2009; 183:2150-2158.

52 Cui J, Zhu L, Xia X, et al. NLRC5 negatively regulates the NF-kappaB and type I interferon signaling pathways. Cell 2010; 141:483-496.

53 Saito T, Hirai R, Loo YM, et al. Regulation of innate antiviral defenses through a shared repressor domain in RIG-I and LGP2. Proc Natl Acad Sci USA 2007; 104:582-587.

54 Kim MJ, Hwang SY, Imaizumi T, Yoo JY. Negative feedback regulation of RIG-I-mediated antiviral signaling by interferon-induced ISG15 conjugation. J Virol 2008; 82:1474-1483.

55 Arimoto K, Takahashi H, Hishiki T, Konishi H, Fujita T, Shimotohno K. Negative regulation of the RIG-I signaling by the ubiquitin ligase RNF125. Proc Natl Acad Sci USA 2007; 104:7500-7505.

56 Saitoh T, Tun-Kyi A, Ryo A, et al. Negative regulation of interferon-regulatory factor 3-dependent innate antiviral response by the prolyl isomerase Pin1. Nat Immunol 2006; 7:598-605.

57 Huang J, Liu T, Xu LG, Chen D, Zhai Z, Shu HB. SIKE is an IKK epsilon/TBK1-associated suppressor of TLR3- and virustriggered IRF-3 activation pathways. EMBO J 2005; 24:40184028.

58 Liu XY, Wei B, Shi HX, Shan YF, Wang C. Tom70 mediates activation of interferon regulatory factor 3 on mitochondria. Cell Res 2010; 20:994-1011.

59 Nakhaei P, Mesplede T, Solis M, et al. The E3 ubiquitin ligase Triad3A negatively regulates the RIG-I/MAVS signaling pathway by targeting TRAF3 for degradation. PLoS Pathog 2009; 5:e1000650.

60 Tseng PH, Matsuzawa A, Zhang W, Mino T, Vignali DA, Karin M. Different modes of ubiquitination of the adaptor 
TRAF3 selectively activate the expression of type I interferons and proinflammatory cytokines. Nat Immunol 2010; 11:70-75.

61 Yoshida R, Takaesu G, Yoshida H, et al. TRAF6 and MEKK1 play a pivotal role in the RIG-I-like helicase antiviral pathway. J Biol Chem 2008; 283:36211-36220.

62 Sun Q, Sun L, Liu HH, et al. The specific and essential role of MAVS in antiviral innate immune responses. Immunity 2006; 24:633-642.

63 Stojdl DF, Lichty B, Knowles S, et al. Exploiting tumorspecific defects in the interferon pathway with a previously unknown oncolytic virus. Nat Med 2000; 6:821-825.

64 Stojdl DF, Lichty BD, tenOever BR, et al. VSV strains with defects in their ability to shutdown innate immunity are potent systemic anti-cancer agents. Cancer Cell 2003; 4:263-275.

65 Servant MJ, Grandvaux N, tenOever BR, Duguay D, Lin R, Hiscott J. Identification of the minimal phosphoacceptor site required for in vivo activation of interferon regulatory factor 3 in response to virus and double-stranded RNA. J Biol Chem 2003; 278:9441-9447.

66 Lin R, Heylbroeck C, Pitha PM, Hiscott J. Virus-dependent phosphorylation of the IRF-3 transcription factor regulates nuclear translocation, transactivation potential, and proteasome-mediated degradation. Mol Cell Biol 1998; 18:29862996.

67 Bibeau-Poirier A, Gravel SP, Clement JF, et al. Involvement of the IkappaB kinase (IKK)-related kinases tank-binding kinase $1 / \mathrm{IKKi}$ and cullin-based ubiquitin ligases in IFN regulatory factor-3 degradation. J Immunol 2006; 177:5059-5067.

68 Gack MU, Shin YC, Joo CH, et al. TRIM25 RING-finger E3 ubiquitin ligase is essential for RIG-I-mediated antiviral activity. Nature 2007; 446:916-920.

69 Hiscott J. Triggering the innate antiviral response through IRF-3 activation. $J$ Biol Chem 2007; 282:15325-15329.

70 Dixit E, Boulant S, Zhang Y, et al. Peroxisomes are signaling platforms for antiviral innate immunity. Cell 2010; 141:668681.

71 Hoepfner D, Schildknegt D, Braakman I, Philippsen P, Tabak HF. Contribution of the endoplasmic reticulum to peroxisome formation. Cell 2005; 122:85-95.

72 Zeng W, Sun L, Jiang X, et al. Reconstitution of the RIG-I pathway reveals a signaling role of unanchored polyubiquitin chains in innate immunity. Cell 2010; 141:315-330.

(Supplementary information is linked to the online version of the paper on the Cell Research website.) 